\title{
Nonadditive changes in conductivity of micro PDLC under the influence of carbon nanotubes and magnetic nanoparticles
}

\author{
P. Kopčansky ${ }^{1}$, M. Timko ${ }^{1}$, Z. Mitrova $^{1}$, V. Zavisova ${ }^{1}$, M. Koneracka $^{1}$, N. Tomašovičova ${ }^{1}$, L. Tomčo $^{1}$, \\ O.V. Kovalchuk ${ }^{2,3}$, V.M. Bykov ${ }^{3}$, T.M. Kovalchuk ${ }^{4}$ \\ ${ }^{1}$ Institute of Experimental Physics, Slovak Academy of Sciences, \\ 47, Watsonova str., 04001 Košice, Slovak Republic \\ ${ }^{2}$ Kyiv National University of Technologies and Design \\ 2, Nemirovich-Danchenko str., Kyiv 01011 \\ ${ }^{3}$ Institute of Physics, National Academy of Science of Ukraine, \\ 46, prospect Nauky, 03650 Kyiv, Ukraine \\ ${ }^{4} V$. Lashkaryov Institute of Semiconductor Physics, National Academy of Science of Ukraine, \\ 45, prospect Nauky, 03650 Kyiv, Ukraine \\ Correspondence authore-mail: akoval@knutd.com.ua
}

\begin{abstract}
Investigated in this work has been the effect of impurities - magnetic nanoparticles (MN) and multiwall carbon nanotubes (MWNT) - separately and together on morphology and dielectric properties of nematic liquid crystal 6CHBT dispersed in polyvinyl alcohol (PDLC). It has been shown that the nanoparticles and nanotubes together change the morphology of PDLC practically in the same manner as every type of impurity singly. The impurities influence also additively on the permittivity at low frequencies and electron component of the conductivity in the polymer matrix. We have found that when MN and MWNT act jointly their ion component of the conductivity exceeds the total changes in conductivity by six times greater than when each type of impurity acts singly. The most probable reason for this nonadditive change in conductivity can be aggregation of these nanoparticles.
\end{abstract}

Keywords: magnetic nanoparticle, multiwall carbon nanotube, permittivity, ion component of the conductivity, electron component of the conductivity, polymer matrix.

Manuscript received 25.06.13; revised version received 28.08.13; accepted for publication 23.10.13; published online 16.12.13.

\section{Introduction}

Carbon multiwall nanotubes (MWNT) occupy a significant place among the nanoparticles that are used to modify the properties of liquid crystals (LC) [1-3]. In $[4,5]$ it has been shown that NT dispersed in liquid crystal can essentially effect on the concentration and spatial distribution of charges and thereby identify regularity of electro-optical response of the composite 'NT - liquid crystal'. In addition, analysis of the concentration dependence of conductivity showed that it can be described as being based on the percolation theory $[1,3,6]$. NT alone cannot always provide the required parameters of LC. For example, to achieve sensitivity to the magnetic field there is a need to introduce magnetic nanoparticles $(\mathrm{MN})$, too $[7,8]$. The question arises whether it is possible, knowing how each impurity acts separately on properties of liquid crystal, to predict the combined effect of nanoparticles at least of two types (MWNT and MN) on the LC properties. In the work [9], we studied this problem for homogeneous liquid crystal. It has been shown that the introduction of NT and MN with the concentration 0.02 wt.\% for each type of nanoparticle into the liquid crystal leads to the almost 20-fold increase in conductivity, while separately each individual nanoparticle increases the conductivity of LC no more than 2 times. These data show that it is difficult to predict the result of collective action even of two types of impurities on LC parameters, if being based on the data on the effect of each impurity separately.

One of the mechanisms, through which explained can be the nonadditive effect of nanoparticles on the LC conductivity detected in this work, can be formation of complexes between the nanoparticles. The size effect (the 
value of the LC volume) can significantly influence on efficiency of this process. It can be realized by dispersing LC molecules in a polymer matrix (PDLC cell).

The purpose of this study was to investigate the influence of carbon nanotubes and magnetic nanoparticles together and singly on the dielectric properties of PDLC for determination of the nonadditive action of these particles (i.e., the effect found for homogeneous LC in [9]).

\section{Materials and methods}

PDLC samples were made as follows. Liquid crystal 6CHBT was introduced into $10 \mathrm{wt} . \%$ aqueous solution of polyvinyl alcohol (PVA). The relation of LC and PVA in mass was amounted as 1:1. The obtained mixture was stirred using a mixer at the frequency $10,000 \mathrm{rpm}$ for 1 min. Then the mixture was deposited on the glass surface or glass surface coated by ITO transparent in the visible spectrum. After water evaporation, the PDLC thin films were obtained. The thickness of the films was $50 \pm 10 \mu \mathrm{m}$. Using the same procedure, the following films were manufactured: films from $\mathrm{MN}$ with the spherical shape and average diameter $5 \mathrm{~nm}$, those from multilayer NT (or MWNT) with the diameter $2 \mathrm{~nm}$ and length $1000 \mathrm{~nm}$ as well as from MN and MWNT together. MN and MWNT were added to a mixture of $\mathrm{LC}$ with an aqueous solution of PVA. The concentration of each nanoparticle with respect to the total weight of LC and PVA was $0.1 \mathrm{wt} . \%$. After stirring, the films were prepared by the same procedure as in the absence of nanoparticles.

The morphology of the obtained films was investigated using the scanning electron microscope JSM-35 (Japan) at the accelerating voltage $35 \mathrm{keV}$. Before doing researches, a thin graphite layer was deposited on the film surface. Thus, all the effects caused by surface charging were minimized. All the obtained images were recorded using a digital camera and then were analyzed.

To investigate the dielectric properties, the PDLC film deposited on ITO was used, where ITO was a lower electrode. Then, additionally, one glass plate coated with ITO was forced to the free PDLC film surface, the glass plate was a top electrode, so the sandwich cell was created. Since the PDLC film comprised only the central part of this cell, by using the interferential method we were able to determine the distance between the electrodes and thus the thickness of the cell. Measurements of the thickness were performed in various places of the sample. This enabled to control the uniformity of attachment of the top electrode. To keep good electrical contact before clamping, the thin LC layer was deposited on the electrode.

Dielectric properties of the obtained sandwich cells were investigated within the frequency range $10^{-1} \ldots 10^{6} \mathrm{~Hz}$ at $293 \mathrm{~K}$ by using the oscilloscopic method [10]. The amplitude of the measuring signal with a triangular shape was $0.25 \mathrm{~V}$.

\section{Result and discussion}

Fig. 1 shows micrographs of the samples: PDLC +0.1 wt. $\%$ MN (a); PDLC + 0.1 wt.\% MWNT (b); PDLC + 0.1 wt. $\% \mathrm{MN}+0.1$ wt. $\%$ MWNT (c). As in the case of PDLC without nanoparticles and PDLC with MN of spherical and elliptical shape considered by us in [11], sizes of LC droplets are 6 to $10 \mu \mathrm{m}$. For further analysis of the results, it is important to compare morphology of the sample shown in Fig. 1c and that of the samples shown in Figs 1a and 1b. As it follows from the analysis of Fig. 1, both in the size of LC droplets and in their concentrations the significant difference between the samples is absent.
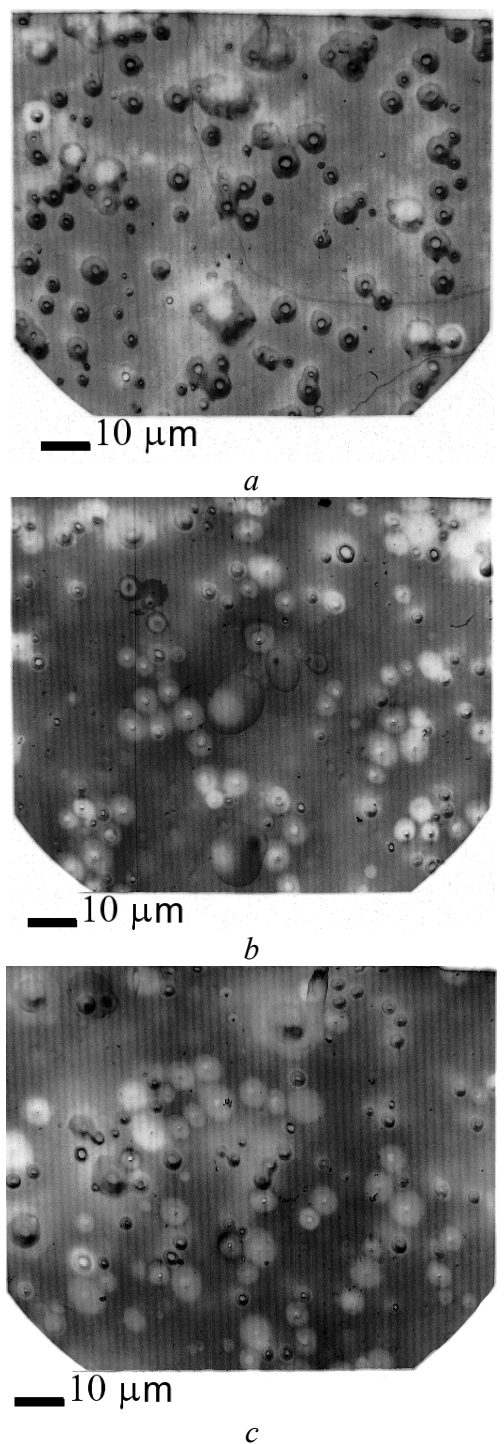

Fig. 1. Morphology of the samples: PDLC $+0.1 \mathrm{wt} . \% \mathrm{MN}(a)$; PDLC +0.1 wt. $\%$ MWNT $(b) ;$ PDLC +0.1 wt. $\%$ MN + 0.1 wt.\% MWNT (c). Images were obtained using the scanning electron microscope JSM-35 (Japan) at the accelerating voltage $35 \mathrm{keV}$. The sample thickness is $50 \pm 10 \mu \mathrm{m}$. The temperature $293 \mathrm{~K}$. 
Fig. 2 shows the frequency dependence of the dielectric constant $\varepsilon$ for the samples : PDLC (1); PDLC + $\quad 0.1 \quad$ wt. $\% \quad$ MN (2); PDLC + 0.1 wt. $\%$ MWNT (3); $\quad$ PDLC + 0.1 wt. $\% \quad$ MN + 0.1 wt. $\%$ MWNT (4). To analyze these results, the whole dielectric spectrum should be separated into two sections $10^{-1} \ldots 10^{3} \mathrm{~Hz}$ and $10^{3} \ldots 10^{6} \mathrm{~Hz}$. The frequency dependence of the $\varepsilon$ value in the first section of the dielectric spectrum is caused by the influence of near-electrode processes [12]. It is just in this section of the dielectric spectrum that the effect of nanoparticles on the $\varepsilon$ value is most essential. Table shows the data for the $\varepsilon$ values for the frequency $0.1 \mathrm{~Hz}$. The analysis of the results shows that the introduction of a small amount of $\mathrm{MN}$ and MWNT (0.1 wt.\%) into PDLC leads to an increase in the $\varepsilon$ value by more than 20 times. Moreover, each of the impurity nanoparticles changes the PDLC $\varepsilon$ value practically identically. When introducing $\mathrm{MN}$ and MWNT together into PDLC, their combined effect on the $\varepsilon$ value does not exceed the total action of each nanoparticle type separately, as it follows from Table. That is it can be asserted that the combined action of $\mathrm{MN}$ and MWNT nanoparticles on the low-frequency $\varepsilon$ value is additive practically with respect to the action of each nanoparticle separately.

In the second section of the dielectric spectrum $10^{3} \ldots 10^{6} \mathrm{~Hz}$, the dielectric properties of the samples are caused by their volume parameters. As follows from Fig. 2, in this section of the spectrum the impact of nanoparticles is much smaller than that in the lowfrequency section. Since the changes in the $\varepsilon$ value, caused by the introduction of nanoparticles, were not much larger than the experimental error, the analysis of data about additivity concerning the action of the nanoparticles was not performed.

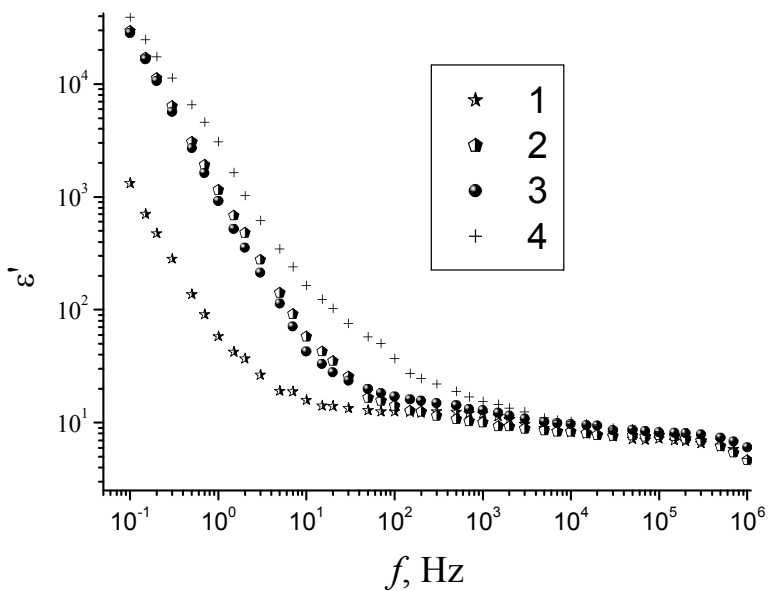

Fig. 2. Frequency dependences of the permittivity $\varepsilon$ for the samples: $\quad$ PDLC (1); PDLC+0.1 wt.\% $\quad \mathrm{MN}(2)$; PDLC+ $0.1 \mathrm{wt} \% \quad$ MWNT (3); PDLC + $0.1 \mathrm{wt} \% \mathrm{MN}+$ 0.1 wt. $\%$ MWNT (4). The sample thickness is $50 \pm 10 \mu \mathrm{m}$. The temperature $293 \mathrm{~K}$.
Table. Dependence of the PDLC parameters on the presence of magnetic nanoparticles and multiwall nanotubes singly and together.

\begin{tabular}{|l|c|c|}
\hline Sample & $\begin{array}{c}\varepsilon \\
f=0.1 \mathrm{~Hz}\end{array}$ & $\begin{array}{c}\sigma_{i}, \\
\mathrm{Ohm}^{-1} \mathrm{~m}^{-1}\end{array}$ \\
\hline PDLC & 1320 & $9.1 \cdot 10^{-9}$ \\
\hline PDLC+01 wt.\% MN & 29550 & $2.2 \cdot 10^{-7}$ \\
\hline PDLC+01 wt.\% MWNT & 28380 & $5.4 \cdot 10^{-5}$ \\
\hline $\begin{array}{l}\text { PDLC+01 wt.\% MN +01 } \\
\text { wt.\% MWNT }\end{array}$ & 39140 & $3.2 \cdot 10^{-4}$ \\
\hline
\end{tabular}

The complexity of this analysis is largely caused by the dependence of $\varepsilon$ value on frequency. For heterogeneous systems, this effect may be caused by the interaction between the particles in the process of their polarization under action of an electric field. In the case of homogeneous liquids such an effect is not observed, and the $\varepsilon$ value is independent of frequency in the highfrequency section of the spectrum.

Fig. 3 shows the frequency dependences of the conductivity $\sigma$ for the following samples: PDLC (1); PDLC + $0.1 \quad$ wt. $\% \quad$ MN (2); PDLC + 0.1 wt. $\%$ MWNT (3); PDLC + $\quad 0.1 \quad$ wt. $\% \quad \mathrm{MN}+\quad 0.1 \quad$ wt. $\%$ MWNT (4). Except for a small decrease of $\sigma$ at most lower frequencies due to the impact of near-electrode processes, the whole dielectric spectrum of conductivity can be separated into two sections.

In the section of frequencies $5-100 \mathrm{~Hz}$, as it follows from Fig. 3, the $\sigma$ value does not depend on the frequency $f$. Independence of conductivity on the frequency is characteristic for the ion conductivity in liquid. In the case of the studied PDLC, this liquid is a liquid crystal. The ion conductivity of PDLC increases significantly in those places of the samples where diameters of droplets are close to the PDLC thickness or in the places of aggregation of drops with slightly smaller diameters than the sample thickness.

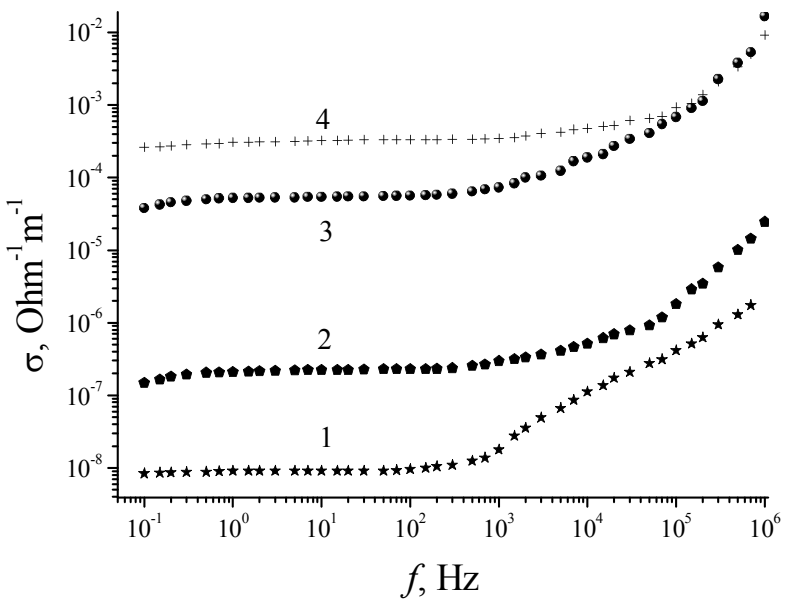

Fig. 3. The frequency dependences of conductivity $\sigma$ for the samples: PDLC (1); PDLC + 0.1 wt.\% MN (2); PDLC + 0.1 wt. $\%$ MWNT (3); PDLC +0.1 wt. $\%$ MN +0.1 wt. $\%$ MWNT (4). The sample thickness is $50 \pm 10 \mu \mathrm{m}$. The temperature $293 \mathrm{~K}$. 
The values of the ion conductivity $\sigma_{i}$ of the studied samples are shown in Table. In contrast to the data concerning the influence of nanoparticles on the $\varepsilon$ value, nonidentical action of MN and MWNT separately on the $\sigma_{i}$ value is observed. So when introducing $0.1 \mathrm{wt} . \% \mathrm{MN}$ into PDLC, the $\sigma_{i}$ value increases by about 25 times; when introducing the same amount of MNT, the $\sigma_{i}$ value increases by more than 6000 times. And this case, the total effect of two types of nanoparticles on condition of additivity should be almost the same as the action of MWNT only separately. In fact, as follows from the table data, the $\sigma_{i}$ value, when introducing $\mathrm{MN}$ and MWNT together, is higher than that when MWNT acts alone.

The formula for calculating the coefficient of conductivity nonadditivity can be written as follows:

$k_{\text {nonad }}=\frac{\sigma_{12}}{\sigma_{1}+\sigma_{2}}$,

where $\sigma_{12}$ is the value of conductivity when acting of both nanoparticle types together; $\sigma_{1}$ and $\sigma_{2}$ are the values of conductivity when each nanoparticle acts separately. The calculation was performed using the Eq. (1) for the Table data, it showed that in the case of the studied samples $k_{\text {nonad }}=6$. For homogeneous samples studied in [9] $k_{\text {nonad }}=5$. In general, one would expect that the value of $k_{\text {nonad }}$ for heterogeneous samples must be less than that for homogeneous ones. The greater $k_{\text {nonad }}$ value for studied samples may be caused by different types of nanotubes, which were used in this work (multiwalled carbon nanotubes) and in the work [9] (singlewall carbon nanotubes).

Considering regularity of the influence of nanoparticles on the conductivity value in the section of the dielectric spectrum $5 \ldots 100 \mathrm{~Hz}$, we shall consider the influence of these impurities on the conductivity value in the section of the dielectric spectrum $10^{3} \ldots 10^{6} \mathrm{~Hz}$. As can be seen from Fig. 3, after the transition section, which has a different frequency range depending on the type of nanoparticles, the conductivity value varies depending on the frequency according to a power law. This type of conductivity is characteristic for disordered materials, and it is associated with intermittent transfer of electrons from one atom (or molecule) to another. In the case of PDLC, the intermittent electron transfer can be carried out through the polymer matrix.

As it follows from Fig. 3, in contrast to ion conductivity, for electron conductivity in polymer the conductivity when introducing MN and MWNT together is practically the same as when introducing MWNT separately. That is the effect of nonadditivity of conductivity change, when the simultaneous introducting two types nanoparticles found for ion component of conductivity for the same samples, is not inherent for the electron component of conductivity. If for explanation of nonadditivity of ion conductivity changes to take into account the assumption about the formation of complexes with different types of nanoparticles, absence of such effect in the case of electron component of conductivity can be easily explained. In the case of a rigid polymer matrix, the efficiency of aggregation of nanoparticles is much lower than that in the liquid phase of LC, because nanoparticles are rigidly fixed in polymer and cannot move as freely as in liquid crystal.

Taking the electron component of conductivity into account, the whole frequency range (except for a small low-frequency section caused by the influence of nearelectrode processes) can be described by the relation

$\sigma=\sigma_{i}+a f^{s}$,

where $a$ is the proportionality coefficient, $s$ is the exponent (for intermittent transfer of electrons $0<s<1)$. It is the ratio between the values $\sigma_{i}$ and $a$ defines the frequency interval for transfer from the section of the dielectric spectrum where the conductivity does not depend on frequency to that where the conductivity is proportional to a power function of frequency .

\section{Conclusions}

From the analysis of microphotos obtained using the scanning electron microscopy, it is seen that the morphology of the films PDLC +0.1 wt. $\% \mathrm{MN}+$ 0.1 wt. $\%$ MWNT slightly differs from that of the films PDLC +0.1 wt. $\% \mathrm{MN}$ and PDLC $+0.1 \mathrm{wt} . \%$ MWNT. That is the combined effect of low concentrations of magnetic nanoparticles and multiwall carbon nanotubes on the morphology of PDLC films is practically the same as that of each impurity separately.

The permittivity at low frequencies (below $10^{3} \mathrm{~Hz}$ ) significantly (by more than an order of magnitude) increases when introducing a small amount $(0.1 \mathrm{wt} . \%)$ of nanoparticles. But in so doing no peculiarities concerning the combined effects of magnetic nanoparticles and multiwall carbon nanotubes to the action of each type of nanoparticles separately are not observed. In other words, we can assume that the change in the permittivity at low frequencies when introducing the magnetic nanoparticles and multiwall carbon nanotubes are additive with respect to the impact of each type of nanoparticle separately.

Except for a small decrease in conductivity at the lowest frequencies of the studied frequency, the dependence of the conductivity of the investigated PDLC films can be separated in two sections. For the section of the frequencies $5 \ldots 100 \mathrm{~Hz}$ the conductivity is independent of frequency, and for the section of the frequencies $10^{3} \ldots 10^{6} \mathrm{~Hz}$ the conductivity is a power function of frequency. The low-frequency section of conductivity is caused by ion conductivity through the liquid crystal, and the high-frequency section of conductivity is caused by the electron one through polymer.

It has shown that the ion component of conductivity when MN and MWNT act jointly exceeds 
the total changes in conductivity by six times greater than when each type of impurity acts singly. One of the most probable causes of this effect may be the formation of complexes between nanoparticles. Confirmation of this hypothesis is the absence of such effect for the electron component of the conductivity. Since aggregation of nanoparticles in polymer occurs less effectively than in the liquid crystal.

\section{References}

1. N. Lebovka, T. Dadakova, L. Lysetskiy et al., Phase transitions, intermolecular interactions and electrical conductivity behavior in carbon multiwalled nanotubes/nematic liquid crystal composites // J. Mol. Struct. 877 (1-3), p. 135-143 (2008).

2. S.-Y Lu, L.-C. Chien, Carbon nanotube doped liquid crystal OCB cells: Dielectric and electrooptical properties // Digest of Technical Papers SID Intern. Sympos. 39(4), p. 1853-1856 (2008).

3. W.Lee, Chun-Yu Wang, Yu-Cheng Shih, Effects of carbon nanosolids on the electro-optical properties of a twisted nematic liquid-crystal host // Appl. Phys. Lett. 85(4), p. 513-515 (2004).

4. A. Koval'chuk, L. Dolgov, and O. Yaroshchuk, Dielectric studies of dispersions of carbon nanotubes in liquid crystals 5CB // Semiconductor Physics, Quantum Electronics \& Optoelectronics, 11(4), p. 337-341 (2008).

5. C-Y. Huang, C-Y Hu, H-C. Pan, and K-Y. Lo, Electrooptical responses of carbon nanotube-doped liquid crystal devices // Jpn. J. Appl. Phys. 44(11), p. 8077-8081 (2005).
6. A.I. Goncharuk, N.I. Lebovka, L.N. Lisetski and S.S. Minenko, Aggregation, percolation and phase transitions in nematic liquid crystal EBBA doped with carbon nanotubes // J. Phys. D: Appl. Phys. 42(16), p.165411-1 - 165411-8 (2009).

7. P. Kopčanský, N. Tomašovičová, M. Koneracká et al.Structural changes in the 6CHBT liquid crystal doped with spherical, rodlike, and chainlike magnetic particles // Phys. Rev. E, 78, 011702 (2008).

8. N. Tomašovičová, P. Kopčanský, M. Koneracká et al., The structural transitions in 6CHBT-based ferronematic droplets // J. Phys.: Condens. Matter, 20(20), 204123 (2008).

9. P. Kopčanský, O.V. Kovalchuk, N. Tomašovičová et al., Nonadditive changes in the conductivity of nematic liquid crystal when introducing singlewalled carbon nanotubes and magnetite // The $13^{\text {th }}$ Small Triangle Meeting on Theoretical Physics. Institute of Experimental Physics, Kosice, Slovakia, p. 72-79 (2012).

10. A.J. Twarowski, A.C. Albrecht, Depletion layer in organic films: Low frequency measurements in polycrystalline tetracene // J. Chem. Phys. 20(5), p. 2255-2261 (1979).

11. P. Kopcansky, M. Timko, Z. Mitrova et al., Morphology and dielectric properties of polymer dispersed liquid crystal with magnetic nanoparticles // Semiconductor Physics, Quantum Electronics and Optoelectronics, 13(4), p. 343-347 (2010).

12. A.V. Koval'chuk, Relaxation processes and charge transport across liquid crystal-electrode interface // J. Phys.: Condens. Matter, 13(46), p. 10333-10346 (2001). 